\title{
Total Video-Assisted Thoracic Surgery Sleeve Lobectomy: Suture by Both Hands
}

\author{
Kai Liu ${ }^{1} \quad$ Chenhua Jin ${ }^{1} \quad$ Hui $\operatorname{Tian}^{1}$ Weiyu Shen ${ }^{1}$ \\ ${ }^{1}$ Department of Thoracic Surgery, Ningbo Medical Center Lihuili \\ Hospital, Ningbo, Zhejiang, China \\ Thorac Cardiovasc Surg Rep 2013;2:43-45.
}

\begin{abstract}
Address for correspondence Hui Tian, MD, Department of Thoracic Surgery, Ningbo Medical Center Lihuili Hospital, Zhejiang Ningbo Xingninglu $57 \mathrm{Hao}$, Ningbo, Zhejiang 315000, China (e-mail: jojo816@163.com).
\end{abstract}

\author{
Abstract \\ Keywords \\ - VATS \\ - sleeve lobectomy \\ - both hands suturing
}

Bronchoplastic procedures offer better long-term survival and quality of life with less morbidity and mortality than pneumonectomies. However, minimally invasive sleeve lobectomy is rarely reported, and the procedure requires more skill even for experienced hands. We report a total video-assisted thoracoscopic right upper sleeve lobectomy for a patient with centrally located lung cancer.

\section{Introduction}

With the development of the techniques of minimally invasive surgery, video-assisted thoracic surgery (VATS) techniques have been increasingly applied to the treatment of lung cancer patients. Total thoracoscopic lobectomy has been performed worldwide, mainly in the lesions that are not centrally located. However, for centrally located lesions, the safety and efficacy of VATS procedures are yet to be determined. We performed a total VATS right upper sleeve lobectomy in a patient with centrally located lung cancer.

\section{Case Report}

A 60-year-old male smoker was referred to our department with "nonsqueezing" chest pain for 1 month. A computed tomographic scan showed a centrally located right upper lesion (-Fig. 1). A biopsy was made with a bronchoscope; the pathology confirmed a non-small cell lung cancer. Preoperative studies and lab results were not notable except for the lung mass.

\section{Operative Technique}

The patient was positioned in a typical left lateral decubitus position and selective intubation was used. The surgeon and the second assistant stood behind the patient. The first assistant stood in front of the patient.

received

June 23, 2013

accepted after revision

August 4, 2013

published online

October 25, 2013
Two 12-mm incisions were made: one in the eighth intercostal space in the midaxillary line, for the camera, and one just below the scapular vertex in the eighth intercostal space; a 5-cm-long anterior minithoracotomy was placed over the fifth intercostal space, without rib spreading, for the insertion of surgical instruments and dissection of vessels and bronchi. The trocar was only introduced to camera port. We used specially designed elongated instruments and endoscopic instruments.

At the beginning of the operation, after confirming the viability of the technique, mediastinal lymph nodes were systematically dissected. Then the lobectomy was performed. After stapling the vessels, the intermediate bronchus was opened and transected, which allowed direct view of the tumor (-Fig. 2). Then the right main bronchus was transected, thus completing the sleeve resection of the tumor including the right upper lobe. The lobe was removed in a glove through the 5-cm-wide incision. This step was performed under full endoscopic view with elongated scissor for open operation. This second specimen was removed in a glove and sent for frozen section, which confirmed free margins.

Bronchial anastomosis was performed in an end-to-end fashion with one running nonabsorbable 3-0 polypropylene (Prolene, Ethicon Inc., Somerville, New Jersey, United States).

The anastomoses started from the membranous portion (posteriorly) and extended to the cartilaginous portion (anteriorly). For the suture of the membranous portion, we used both hands: first the needle holder was placed by the right
License terms

Stuttgart · New York

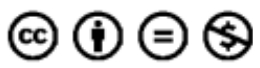




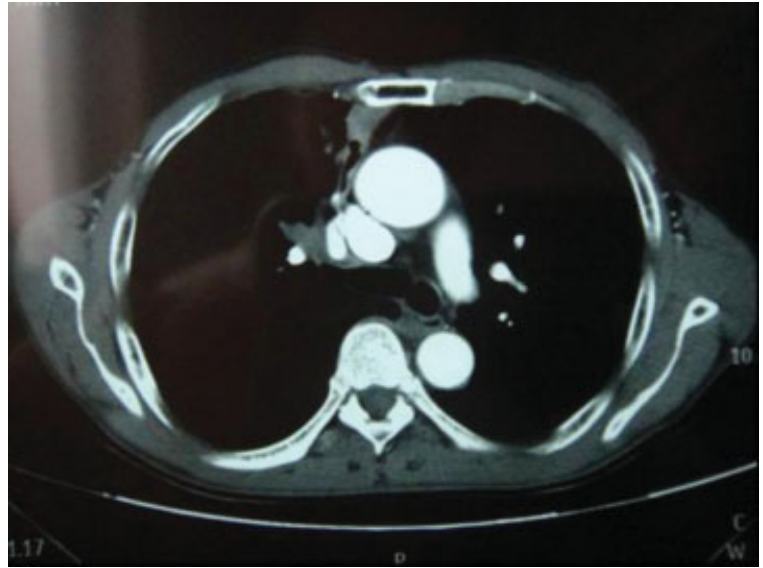

Fig. 1 A computed tomographic scan of the 60-year-old man showing a centrally located right upper lesion.

hand through the 5-cm-wide incision to suture the proximal end from inside (-Fig. $\mathbf{3}$ ). Then to suture the distal end, the needle holder was transferred to the back incision in the eighth intercostal space, and the suture was placed in the distal end by the left hand from outside (-Fig. 4). After completing the posterior suture, the anterior was sutured through the main access port (the minithoracotomy) in traditional fashion. As the running suture was completed, we used the endoscopic coagulator to retract the suture to ensure the proper tension. After the suture was tied, the anastomosis was completed (-Fig. 5).

Finally, the anastomosis was sealed under saline, and $20 \mathrm{~mm} \mathrm{Hg}$ ventilation positive end-expiratory pressure was set; no air leak was detected.

Intraoperative blood loss was minimal, and total operative time was 240 minutes, including 65 minutes for performing the anastomosis.

During the operation, the patient had onset atrial fibrillation. Amiodarone was deployed, and the heart rate was controlled immediately. The patient was extubated in the operating room and brought to recovery and eventually

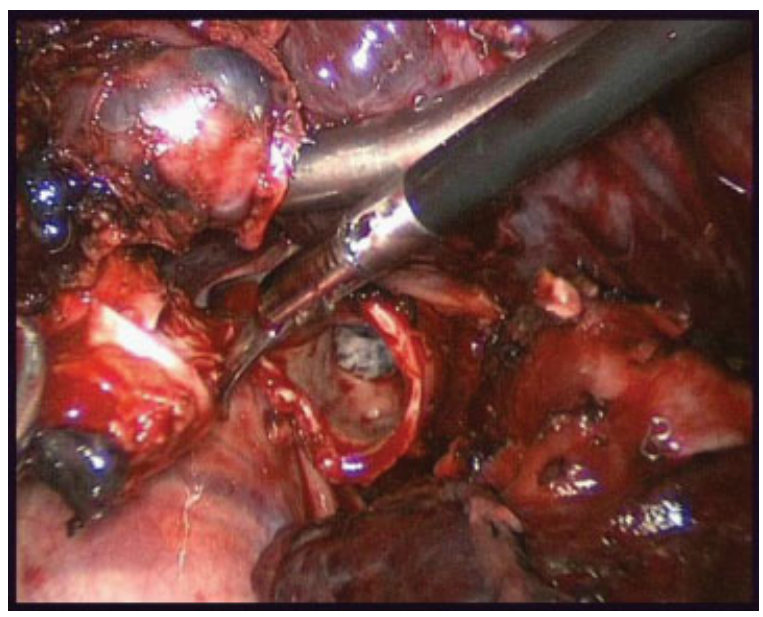

Fig. 2 Cutting of the proximal bronchus by endoscissors.

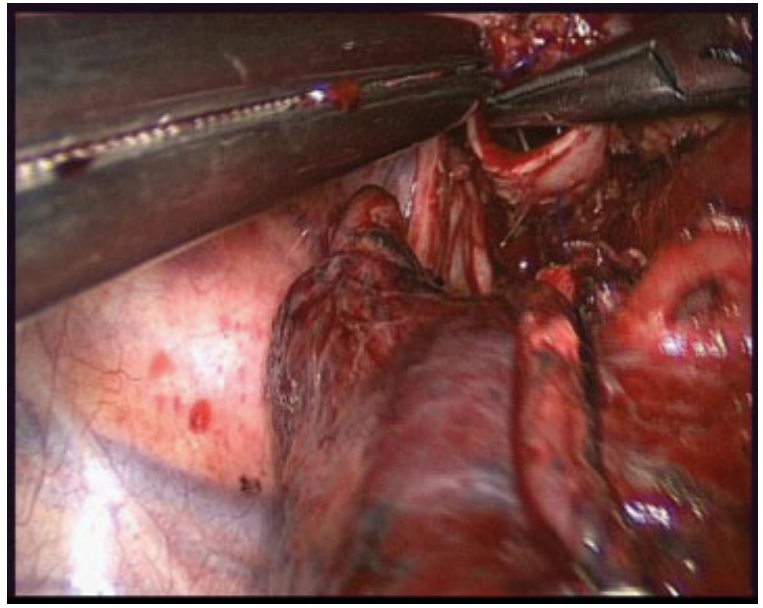

Fig. 3 Suture of the anastomosis by the right hand.

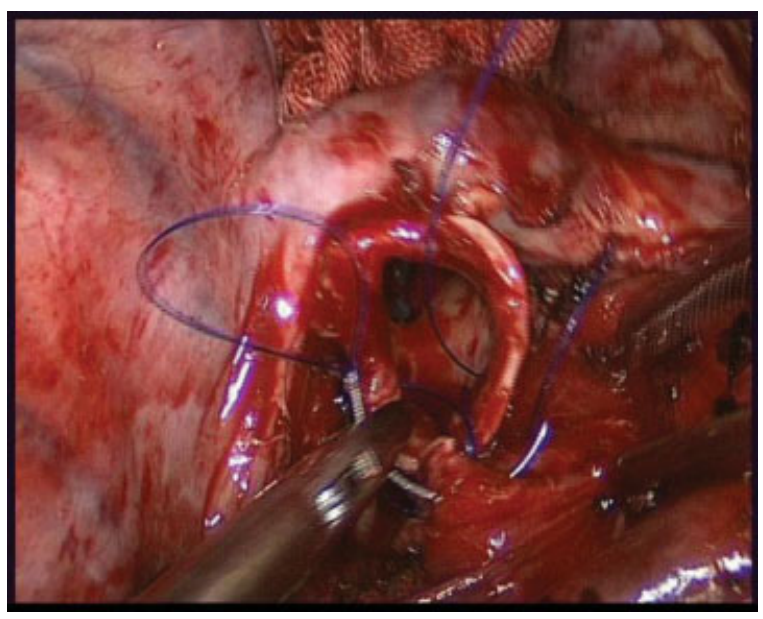

Fig. 4 Suture of the anastomosis by the left hand.

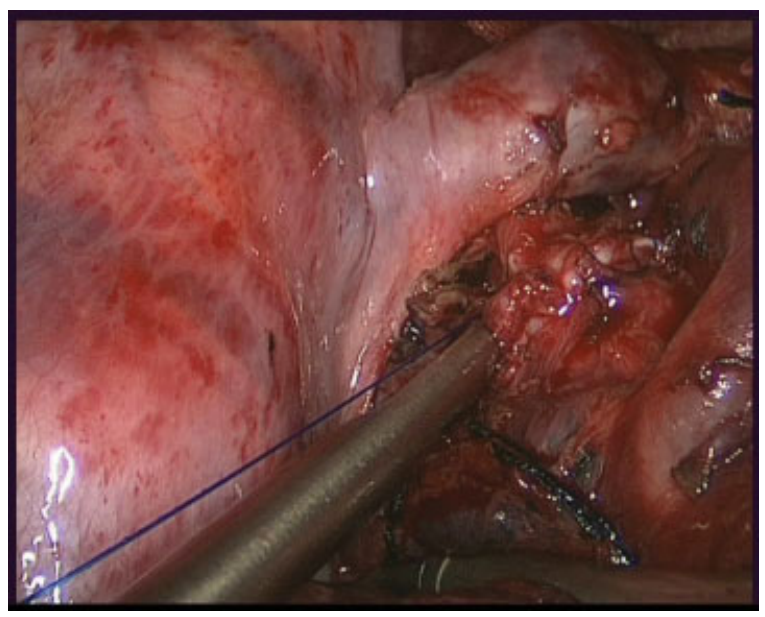

Fig. 5 Completion of the anastomosis. 
moved to the regular floor. On third postoperative day, the heart returned to sinus rhythm. The chest tube was removed on the fourth postoperative day. The patient was discharged on the 10th postoperative day.

The pathology confirmed a poorly differentiated squamous lung cancer, with $\mathrm{N} 1$ lymph node positive (pT1apN1M0). The patient received three cycles of adjuvant chemotherapy after discharge. The patient remained in good health until March 2013 when he received another VATS left upper lobectomy for adenocarcinoma (pT1apNOM0). The patient tolerated the second operation very well and was discharged on the fifth postoperative day.

\section{Discussion}

Sleeve lobectomy was designed to treat low-grade lung malignancy.

In current studies, without compromising the oncological radicality, bronchoplastic procedures offer better long-term survival and quality of life with less morbidity and mortality than pneumonectomies. The increasing number of clinical series in the literature indicates that bronchoplastic procedures have been widely adopted worldwide and should be considered in any case of lung cancer that can be completely resected using these procedures. ${ }^{1,2}$

However, the procedure requires skill, and minimally invasive sleeve lobectomy is more challenging even for experienced hands. A few cases have been reported. ${ }^{3-5}$ Total VATS sleeve lobectomy seems to be more cost-effective than a hybrid procedure.

With increased experience with VATS, we performed this operation. We realized VATS sleeve lobectomy is a very different procedure, owning to the confined costal space for surgical access. During the operation, the suture is conducted endoscopically. Traditional hand-eye coordination is re- placed by hand movements conducted by a two-dimensional visual system; we found traditional way of suturing timeconsuming when obtaining a proper needle position. Therefore, we modified the technique during suture of the membranous part. The surgeon felt more comfortable about both hands suturing, which accelerated the process of the procedure.

The uncomplicated minimally invasive sleeve lobectomy allowed this patient to tolerate the operation for the second malignancy very well, which may not have been possible if pneumonectomy or conventional procedure had been performed.

In conclusion, minimally invasive sleeve lobectomy is safe and feasible, with proper selection of potential candidates. The suture technique under thoracoscopy may differ from that in open surgery. Furthermore, a large case series may be required to prove our results.

\section{References}

1 Rea F, Marulli G, Schiavon M, et al. A quarter of a century experience with sleeve lobectomy for non-small cell lung cancer. Eur J Cardiothorac Surg 2008;34(3):488-492, discussion 492

2 Deslauriers J, Grégoire J, Jacques LF, Piraux M, Guojin L, Lacasse Y. Sleeve lobectomy versus pneumonectomy for lung cancer: a comparative analysis of survival and sites or recurrences. Ann Thorac Surg 2004;77(4):1152-1156, discussion 1156

3 Santambrogio L, Cioffi U, De Simone M, Rosso L, Ferrero S, Giunta A. Video-assisted sleeve lobectomy for mucoepidermoid carcinoma of the left lower lobar bronchus: a case report. Chest 2002;121 (2):635-636

4 Mahtabifard A, Fuller CB, McKenna RJ Jr. Video-assisted thoracic surgery sleeve lobectomy: a case series. Ann Thorac Surg 2008;85 (2):S729-S732

5 Schmid T, Augustin F, Kainz G, Pratschke J, Bodner J. Hybrid videoassisted thoracic surgery-robotic minimally invasive right upper lobe sleeve lobectomy. Ann Thorac Surg 2011;91(6):1961-1965 